\title{
Timing of pulp extirpation for replanted avulsed teeth
}

\author{
Abstracted from \\ Hinckfuss SE, Messer LB. \\ An evidence-based assessment of the clinical guidelines for replanted avulsed teeth. \\ Part I. Timing of pulp extirpation. Dent Traumatol 2009; 25: 32-42 \\ Address for correspondence: Professor Louise Brearley Messer, School of Dental Science, \\ Faculty of Medicine, Dentistry and Health Sciences, University of Melbourne, 720 Swanston Street, \\ Victoria 3010, Australia. E-mail: ljbm@unimelb.edu.au
}

Question: How long should we wait for a pulp extirpation after a replantation of an avulsed permanent tooth?

Data Sources A search was performed (April 2004) across four databases, namely Ovid Medline, Cochrane Library, PubMed and Web of Science, relevant to the proposed PICO (Patient or problem, Intervention, Comparison, Outcome) question: $(P)$ for a replanted avulsed permanent tooth, (I) is early pulp extirpation within 10-14 days of replantation, (C) compared with delayed pulp extirpation, $(\mathrm{O})$ associated an increased likelihood of successful periodontal healing after tooth replantation. Only articles published in the English language were considered.

Study selection Relevant titles were selected for abstract assessment $(\mathrm{N}=628)$, and then 84 papers were selected for examination.

Data extraction and synthesis A quality assessment was made of relevant publications: only six papers met the inclusion criteria (making a total of 236 teeth).

Results Meta-analyses found a statistically significant association between pulp extirpation performed after 14 days and the development of inflammatory resorption. Pulp extirpation within 10 days of replantation was not significantly associated with a decreased likelihood of developing inflammatory resorption. There is no significant difference in pulp extirpation times for functional healing, acceptable healing without progressive resorption, or the development of replacement resorption. Conclusions There is clinical evidence for an association between pulp extirpation performed after 14 days following replantation and the development of inflammatory resorption. This investigation supports the current clinical guidelines for pulp extirpation within 10-14 days of replantation.

\section{Commentary}

The timing of pulp extirpation in avulsed replanted incisors is of concern to clinicians faced with the management of dental avulsion. The perceived benefits of early intervention to extirpate pulp must be balanced against the need to allow time for initial injury healing, involving hard and soft tissues and resolution of associated pain and swelling. Existing guidelines draw on evidence from available studies in the literature including valuable early studies in animals. The nature of dento-alveolar trauma dictates the structure of human studies, and the timing of interventions is often influenced by circumstances outside the control of the clinician.

The authors here endeavour to identify and draw together relevant studies that relate specifically to the timing of pulp extirpa- tion in replanted avulsed teeth and to associate the intervention with desired outcomes. A well-structured search of the literature is described, and application of strict exclusion/ inclusion criteria resulted in the selection of six studies that dealt with timing of pulp extirpation and periodontal healing outcomes. The PICO research question is presented, giving focus to the objectives. Although the intervention phrase, "early pulp extirpation within 10 to 14 days of replantation" might suggest that only pulp extirpation occurring between these times is considered, the text clarifies early $\mathrm{PE}$ as occurring up to 10 days in some studies and up to 14 days in others, ie, extirpation prior to 10 days is included in both instances.

The authors reclassified periodontal healing outcomes from the six studies to functional, acceptable healing and replacement or inflammatory resorption. Their analysis determined that the timing of pulp extirpation influenced the occurrence of inflammatory resorption. No relationship was found with the other outcomes.

These authors acknowledge the limitations of such an investigation, including the influence of confounding factors in injury and patient factors. Other limitations include the lack of standardised recording methodology between the six studies, three of which were prospective and three retrospective. No differentiation was made between teeth with open apices and those with closed apices in some of the data included. Statistical meta-analysis is used to smooth out some of the heterogeneity and the conclusions must be considered in this context. Despite the limitations described by the authors, the review appears to be valid and the study results analysed are sufficiently robust to produce a significant result. The conclusion of this investigation that the likelihood of successful periodontal healing is improved if pulp extirpation occurs within 14 days, because of decreased inflammatory resorption, supports existing guidelines.

\section{Practice point \\ - For replanted avulsed teeth with closed apices, elective extirpation of the pulps should be performed within 14 days. For teeth with open apices, pulps should be extirpated when there is evidence of pulpal necrosis. \\ - Pulpal extirpation is beneficial in the control of inflammatory resorption but has no proven benefit in control of replacement resorption or other aspects of healing. \\ Chris Stewart \\ Dept Paediatric Dentistry, Cork University Dental School and Hospital, Cork, Ireland.}

Evidence-Based Dentistry (2009) 10, 72. doi:10.1038/sj.ebd.6400664 